\title{
Michael Krennerich
}

\section{Die Universalisierung der Menschenrechte - die rechtlichen und politischen Dimensionen im Fokus}

Menschenrechte sind mit dem Anspruch verbunden, universell zu gelten. Über Traditionen und kulturelle Eigenheiten hinweg fixieren sie einen Grundbestand an Rechten, der für jeden Menschen gelten soll. Dies meint das Prinzip der Universalität der Menschenrechte. Der Geltungsanspruch bezieht sich dabei nicht nur darauf, dass die Menschenrechte weltweit, also global gelten sollen, sondern hebt auch auf den Charakter der Menschenrechte als gleiche Rechte für jeden Menschen ab. Insofern sind auch die in den meisten regionalen Menschenrechtsabkommen niedergelegten Rechte, sofern sie auf dem Prinzip der gleichen Achtung der Menschen beruhen, ihrem Wesen nach als universelle Menschenrechte gedacht oder konzipiert, auch wenn sie territorial nicht für alle Menschen Geltung beanspruchen. Der Gegenbegriff zu Universalität ist demnach Partikularität.

Angesiedelt zwischen Moral, Politik und Recht, sind Menschenrechte komplexe Rechte. Sie lassen sich moralisch begründen und werden durch politische Entscheidungen zu positiven Rechten, die in der modernen Staatenwelt in internationalen Menschenrechtsabkommen sowie im Idealfall in Grundrechtskatalogen nationaler Verfassungen verankert sind. Diese weisen die einzelnen Menschen als Trägerinnen und Träger der Menschenrechte aus und verpflichten vornehmlich (aber nicht nur) die Staaten dazu, die Menschenrechte zu achten, zu schützen und zu gewährleisten. Mit Blick auf die Universalität der Menschenrechte ist daher deren moralische, rechtliche und politische Geltung zu unterscheiden. Obwohl alle drei Bereiche zusammenhängen, folgen sie unterschiedlichen Argumentations- und Handlungsmodi.

\section{Die moralische Begründung - der Kern der Universalitätsdebatte}

Die Frage nach der moralischen Begründung und Geltung der Menschenrechte steht traditionell im Zentrum der Debatte um deren Universalität. ${ }^{1}$ Es handelt sich zunächst um eine theoretisch-philosophische Frage, geht es doch darum, hinreichend verallgemeinerbare Rechtfertigungsgründe für den universellen Geltungsanspruch der Menschenrechte zu finden. Bereits die Vielzahl konkurrierender Begründungsversuche und Ausdeutungen der Menschenrechte im westlichen Diskurs zeigt freilich, dass die Menschenrechte unterschiedlichen Begründungen und Interpretationen zugänglich sind, von anthropologischen und essentialistischen über vernunft-, vertrags- und diskurstheoretische bis hin zu religiösen. ${ }^{2}$ Dieser Eindruck wird im interkulturellen Diskurs noch verstärkt. 
Der vorliegende Beitrag beteiligt sich nun nicht an der philosophischen Debatte um die moralische Begründung und Interpretation der Menschenrechte, sondern wendet sich vornehmlich deren rechtlichen und politischen Geltung und »Universalisierung « zu. Dennoch sei eine Vorbemerkung zur praktischen Bedeutung der normativen Diskussion erlaubt. Während die Vielbegründbarkeit vor allem für jene Autoren ein Problem darstellt, welche den Universalitätsanspruch in einer absoluten Weise zu begründen versuchen, ist die Begründungsvielfalt für die tatsächliche Anerkennung der Menschenrechte nicht unbedingt von Nachtteil: So müssen Menschenrechtsnormen nicht überall mit der gleichen, sondern können auch mit unterschiedlichen Begründungen anerkannt werden. In diesem Sinne empfiehlt beispielsweise Abdullahi Ahmed An-Na'im die Universalität der Menschenrechte nicht nur säkular, sondern auch religiös zu begründen, da man so auch gläubige Menschen dazu bewegen könne, sich für die Menschenrechte einzusetzen. ${ }^{3}$

Um die Vielbegründbarkeit für den interkulturellen Diskurs fruchtbar zu machen, ist es freilich vonnöten, die Genese und die Begründung der Menschenrechte voneinander zu trennen. Die Tatsache, dass die Menschenrechte in ihrer heutigen Form historisch in Nordamerika und Europa zum Durchbruch kamen und in mancherlei Hinsicht - etwa in Bezug auf die herausgehobene Bedeutung individueller Selbstbestimmung und die Rolle des Staates - einen »kulturellen bias « aufweisen, bedeutet nicht, dass sie nicht auch unter anderen Bedingungen in anderen Weltregionen zum Durchbruch kommen können und zwangsläufig auf die ideengeschichtlichen Begründungen des »Westens « angewiesen wären. Die Menschenrechte lassen auch andere Entstehungskontexte und andere Begründungszusammenhänge zu. Die westliche Begründung und Durchsetzung der Menschenrechte bieten daher lediglich ein - wenn auch prominentes und prägendes - Beispiel, wie die Idee der Menschenrechte begründet und verwirklicht werden kann. ${ }^{4}$ Im interkulturellen Vergleich und Dialog ist daher herauszuarbeiten, welche und möglicherweise auch welche anderen, »nicht-westlichen« Begründungen herangezogen werden können, um die Menschenrechte auf eine normativ-philosophische Grundlage zu stellen. Al-

1 Vgl. Ludger Kühnhardt, Die Universalität der Menschenrechte, München 1987; Heiner Bielefeldt, Philosophie der Menschenrechte, Darmstadt 1998; Raúl Fornet-Betancourt (Hg.), Menschenrechte im Streit zwischen Kulturpluralismus und Universalität, Frankfurt a.M. 2000; Matthias König, »Universalismus und Relativismus - Zur Begründbarkeit der Menschenrechte« in: Siegfried Frech / Michael Haspel (Hg.), Menschenrechte, Schwalbach/Ts. 2005, S. 91-112; Christoph Menke / Arnd Pollmann: Philosophie der Menschenrechte zur Einfübrung, Hamburg 2007; Günter Nooke / Georg Lohmann / Gerhard Wahlers (Hg.), Gelten Menschenrechte universal? Begründungen und Infragestellungen, Freiburg/Basel/Wien 2008.

2 Vgl. etwa Robert Alexy, »Menschenrechte ohne Metaphysik « in: Deutsche Zeitschrift für Philosophie, Jg. 52, H. 1 (2004), S. 15-24, sowie den Beitrag von Arnd Pollmann in dieser Zeitschrift.

3 Abdullahi Ahmed An-Na’im, »Der Islam, die Menschenrechte und der Säkularismus« in: böll Thema, Nr. 3 (2008), S. 33 f.

4 Erfreulich deutlich: Bielefeldt, Philosophie der Menschenrechte, aaO. (Fn. 1); ders., »Menschenrechtlicher Universalismus ohne eurozentrische Verkürzung « in: Nooke / Lohmann / Wahlers, Gelten Menschenrechte universal?, aaO. (Fn. 1), S. 98-141. 
lerdings ist dabei die gleiche Achtung der Menschen ein unhintergehbarer Grundanspruch und Bestandteil der Menschenrechte in ihrer Eigenschaft als universelle Menschenrechte.

Eine zugrunde liegende, oft stillschweigende Annahme der normativen Debatte um die Universalität der Menschenrechte lautet hierbei: Menschen erkennen die Menschenrechte (nur) dann an, wenn sie auch deren jeweiligen Geltungsansprüche anerkennen. Insofern kommt der Begründung der Menschenrechte grundlegende Bedeutung für ihre faktische Akzeptanz zu. Soll die Universalität der Menschenrechte im normativen Sinne befördert und vermittelt werden, sind daher insbesondere die Handlungsmodi argumentativen und kommunikativen Handelns gefragt: die kritische Reflexion bestehender Traditionen, Werte und Normen, die Hinterfragung expliziter und impliziter Geltungsgründe des Denkens und Handelns, das Herausarbeiten von normativem Konsens und Dissens, Diskurs und interkulturelle Verständigung sowie Kritik, Überzeugung und Bereitschaft, sich selbst überzeugen zu lassen. Die Frage, welche Überzeugungskraft »besseren Argumenten« für die Anerkennung der Menschenrechte tatsächlich zukommt, ist dabei eigens zu prüfen.

Einschränkend ist darauf hinzuweisen, dass in der Praxis keine idealen Voraussetzungen für einen offenen Diskurs bestehen, in dem normative Geltungsansprüche auf den Prüfstand und zur Diskussion gestellt werden, schon gar nicht in der Politik. Sowohl die internationalen als auch die nationalen politischen Diskurse weisen für gewöhnlich stark macht- und interessengeleitete, mithin hegemoniale Züge und eine unzureichende Verständigungsorientierung auf. Bestenfalls lassen sich »Inseln der Überzeugung " ausmachen. ${ }^{5}$

Selbst dort, wo ein Verständigungswille besteht, wird das eigene und fremde Handeln und Denken meist nicht auf die zugrunde liegenden normativen Geltungsansprüche und -gründe zurückgeführt. Eine solche grundlegende Reflexion ist zwar das Geschäft philosophisch geschulter Autoren. Diese müssen aber wohl oder übel damit leben, dass die Überzeugungskraft der Menschenrechte de facto weniger auf ausgefeilten moralischen Begründungen als auf der - philosophisch gewiss unbefriedigenden ${ }^{6}$ - Evidenz kontingenter Erfahrungen von Gewalt, Unterdrückung und Ausbeutung beruht.

Die Universalisierung der Menschenrechte hängt, so betrachtet, auch davon ab, $\mathrm{ob}$ und inwieweit historisch und kulturell geprägte Gewalt- und Unrechtserfahrungen der Menschen inter- und intrakulturell nachvollziehbar sind - und welche politischen Lehren daraus gezogen werden. Die in der Allgemeinen Erklärung der Menschenrechte (AEMR) von 1948 verankerten Rechte lassen sich hierbei als allgemeine

5 Der Begriff ist entlehnt aus: Nicole Deidelhoff, Überzengung in der Politik, Frankfurt a.M. 2007.

6 Thomas M. Schmidt hält beispielsweise Begründungsformen, die auf historische Erfahrungen rekurieren, als »zu niedrig « angesetzt, da sie auf etwas Partikularem und Kontingentem beruhen; vgl. Thomas M. Schmidt: »Diskursive Verpflichtungen und ethischreligiöse Überzeugungen. Zur moralischen und politischen Geltung der Menschenrechte" in: Nooke / Lohmann / Wahlers, Gelten Menschenrechte universal?, aaO. (Fn. 1), S. 142-164, hier: S. 145. 
Antworten auf konkrete historische Erfahrungen und zugleich als Ausdruck historischer Emanzipationsbestrebungen verstehen. Als solche werden sie unter den jeweiligen Bedingungen vor Ort immer wieder neu aufgegriffen, aktualisiert und kulturbezogen gedeutet. ${ }^{?}$

\section{Die Universalisierung der Menschenrechte aus rechtlicher Sicht}

Aus rechtlicher Perspektive ist für die Universalisierung der Menschenrechte bedeutsam, dass sich internationale Organisationen zum Ziel der Achtung der Menschenrechte bekennen und die Menschenrechte in völkerrechtlichen Verträgen kodifiziert sind. Mit der Mitgliedschaft in solchen Organisationen und der (vorbehaltlosen) Ratifizierung internationaler Menschenrechtsabkommen verpflichten sich die Staaten dazu, die Menschenrechte umzusetzen. Dabei ist zu klären, inwieweit sich auf internationaler Ebene zumindest ein grundlegender rechtsdogmatischer Konsens über den Inhalt, die Schranken und das Verhältnis der Menschenrechte zueinander herausgebildet hat. Darüber hinaus ist wichtig, dass die Einhaltung der Menschenrechte institutionell abgesichert wird und sich die Staaten entsprechenden Überwachungs- und Durchsetzungsmechanismen unterwerfen. Von herausragender Bedeutung ist schließlich, ob die Menschenrechte in den jeweiligen nationalen Rechtsordnungen verankert sind.

\subsection{Die Kodifizierung und Anerkennung internationaler Menschenrechte}

Hinsichtlich der Kodifizierung internationaler Menschenrechte wurden in den vergangenen 60 Jahren erhebliche Fortschritte erzielt. Die - ursprünglich nicht rechtlich bindende - Allgemeine Erklärung der Menschenrechte gilt nach überzeugendem Rechtsverständnis mittlerweile als die autorisierte Auslegung des menschenrechtlichen Auftrags der UN-Charta, und ihre Bestimmungen sind größtenteils zum Völkergewohnheitsrecht erstarkt. ${ }^{8}$ Mit wenigen Ausnahmen wurden zudem die Rechte der AEMR bekanntlich in zwei völkerrechtlich verbindliche Menschenrechtsabkommen überführt, namentlich den Internationalen Pakt über die bürgerlichen und politischen Rechte (UN-Zivilpakt) sowie den Internationalen Pakt über die wirtschaftlichen, sozialen und kulturellen Rechte (UN-Sozialpakt). Gemeinsam mit der Allgemeinen Erklärung bilden die beiden UN-Pakte die Grundlage einer Vielzahl weiterer universeller Menschenrechtsabkommen, die einzelne Rechte oder gefährdete Personengruppen besonders schützen sollen. Teils parallel zur Kodifizierung internationaler Menschenrechtsnormen auf der Ebene der

7 Vgl. Rainer Huhle / Michael Krennerich, »Die Allgemeine Erklärung der Menschenrechte: eine grundlegende Antwort auf historisches Unrecht « in: transit. Zeitschrift für Politik und Zeitgeschichte, Nr. 2 (2008), S. 4-10.

8 Statt vieler: Eibe Riedel, "Der internationale Menschenrechtsschutz. Eine Einführung « in: Bundeszentrale für Politische Bildung (Hg.), Menschenrechte. Dokumente und Deklarationen, Bonn 2004, S. 11-40, hier: S. 15. 
Vereinten Nationen oder auch der Internationalen Arbeitsorganisation wurden zudem in Europa, Amerika und Afrika regionale Menschenrechtsschutzsysteme entwickelt. Hinzu kommen das Humanitäre Völkerrecht und das sich in den vergangenen Jahren dynamisch entwickelnde internationale Strafrecht. ${ }^{9}$

\begin{tabular}{|l|l|l|}
\hline Abkommen & $\begin{array}{l}\text { verabschiedet/ } \\
\text { in Kraft }\end{array}$ & $\begin{array}{l}\text { Ratifi- } \\
\text { kationen }\end{array}$ \\
\hline $\begin{array}{l}\text { Internationales Übereinkommen zur Beseiti- } \\
\text { gung jeder Form von Rassendiskriminierung } \\
\text { (ICERD) }\end{array}$ & $1965 / 1969$ & 173 \\
\hline $\begin{array}{l}\text { Internationaler Pakt über bürgerliche und } \\
\text { politische Rechte (ICCPR) }\end{array}$ & $1966 / 1976$ & 163 \\
\hline $\begin{array}{l}\text { Internationaler Pakt über wirtschaftliche, } \\
\text { soziale und kulturelle Rechte (ICESCR) }\end{array}$ & $1966 / 1976$ & 159 \\
\hline $\begin{array}{l}\text { Übereinkommen zur Beseitigung jeder Form } \\
\text { der Diskriminierung der Frau (CEDAW) }\end{array}$ & $1979 / 1981$ & 185 \\
\hline $\begin{array}{l}\text { Übereinkommen gegen Folter und andere } \\
\text { grausame, unmenschliche oder erniedrigende } \\
\text { Behandlung oder Strafe (CAT) }\end{array}$ & $1984 / 1987$ & 146 \\
\hline $\begin{array}{l}\text { Übereinkommen über die Rechte des Kindes } \\
\text { (CRC) }\end{array}$ & $1989 / 1990$ & 193 \\
\hline $\begin{array}{l}\text { Internationales Übereinkommen zum Schutz } \\
\text { der Rechte aller Wanderarbeitnehmer und } \\
\text { ihrer Familien (ICRMW) }\end{array}$ & $1990 / 2003$ & 40 \\
\hline $\begin{array}{l}\text { Übereinkommen über die Rechte von } \\
\text { Menschen mit Behinderungen (ICRPD) }\end{array}$ & $2006 / 2008$ & 45 \\
\hline
\end{tabular}

Quelle: www.ohchr.org (Stand: 27.12.2008)

Tabelle 1: Kernabkommen des UN-Menschenrechtsschutzes (ohne Zusatzprotokolle)

Aus völkerrechtlicher Perspektive mutet die philosophische Debatte um die Universalität der Menschenrechte auf den ersten Blick befremdlich an, denn die derzeit gültigen internationalen Abkommen beinhalten weit mehr Menschenrechte als Philosophen gemeinhin für universell begründbar halten; sie wurden dennoch von der großen Mehrzahl der Staaten ratifiziert, und zwar von Staaten ganz unterschiedlicher politischer, kultureller und religiöser Prägung. Spitzenreiter ist hier die UNKinderrechtskonvention, die weltweit nur von Somalia und den USA nicht ratifiziert wurde. Insgesamt wuchs die Zahl der Vertragsstaaten internationaler Men-

9 Zum Überblick: Walter Kälin / Jörg Künzli, Universeller Menschenrechtsschutz, Basel/ Genf/München 2005. 
schenrechtsabkommen in den vergangenen beiden Jahrzehnten erheblich, und es gibt nur noch wenige Staaten, die keinen der beiden grundlegende Pakte, also weder den UN-Sozialpakt noch den UN-Zivilpakt, ratifiziert haben. Dazu gehören neben verschiedenen Inselstaaten der Karibik, des Pazifiks und Afrikas ${ }^{10}$ nur einige Staaten Süd(ost)asiens (Bhutan, Brunei, Malaysia, Myanmar, Singapur) und des Nahen Ostens (Katar, Oman, Saudi-Arabien, Vereinigte Arabische Emirate).$^{11}$ Lediglich ein halbes Dutzend Staaten ist zudem Vertragspartei nur eines der beiden Pakte. Dazu zählen allerdings die USA, die mit Ausnahme des UN-Zivilpakts und der Konvention gegen die Rassendiskriminierung ohnehin kein universelles Kernabkommen ratifiziert haben, sowie China, dessen Ratifikation des UN-Zivilpakts und der in dem Land besonders wichtigen Wanderarbeiterkonvention noch aussteht.

Angesichts der gewohnheitsrechtlichen Bindungskraft der AEMR und des mittlerweile hohen Ratifikationsstandes internationaler Menschenrechtsabkommen scheint die Universalisierung der Menschenrechte völkerrechtlich weit vorangeschritten zu sein. Immerhin wurden die Menschenrechtsverträge in souveränen Entscheidungen der Staaten angenommen, sind völkerrechtlich verbindlich und gemäß dem Grundsatz "pacta sunt servanda" auch einzuhalten.

\subsection{Ein gemeinsames Grundverständnis der Menschenrechte?}

Beim näheren Hinschauen sind freilich verschiedene Einschränkungen vonnöten. Trotz aller Abkommen werden einzelne Menschenrechtsgarantien - wie etwa die Gleichberechtigung von Mann und Frau oder das Recht, die Religion aufzugeben unter Berufung auf religiöse Gebote oder kulturelle Traditionen immer wieder in Frage gestellt. ${ }^{12}$ Auch bringen die Staaten vielfach formale Vorbehalte gegenüber einzelnen Menschenrechtsnormen bzw. -abkommen vor. Die Liste der sog. reservations zu den Verträgen ist lang.

$\mathrm{Da}$ die Menschenrechte ihrer Natur nach allgemein formuliert und auslegungsbedürftig sind, kann zudem kein gemeinsames Grundverständnis von den Menschenrechten und den sich daraus ergebenden Pflichten vorausgesetzt werden. Obwohl die Auslegung der Menschenrechte - auch angesichts entsprechender Vorgaben internationaler Menschenrechtsorgane ${ }^{13}$ - mitnichten beliebig ist, gibt es noch große Interpretationsunterschiede bezüglich der Menschenrechte im Allgemeinen sowie der einzelnen Menschenrechtsnormen und ihres Verhältnisses zueinander. So ist gerade im interregionalen Diskurs umstritten, wie sich die einzelnen Rechte und ihre materiellen Schutzbereiche inhaltlich konkretisieren lassen und wann eine Menschenrechtsverletzung vorliegt.

10 Etwa Antigua und Barbuda, Kuba*, St. Kitts und Nevis, St. Lucia; Fidschi, Kiribati, Marshallinseln, Mikronesien, Palau, Tonga, Tuvalu; Komoren*, São Tomé und Príncipe* (" gezeichnet, aber nicht ratifiziert).

$11 \mathrm{Zu}$ den Ratifikationsständen siehe: www.ohchr.org.

12 Vgl. Kälin / Künzli, Universeller Menschenrechtsschutz, aaO. (Fn. 9), S. 22.

13 Vgl. etwa: Deutsches Institut für Menschenrechte (Hg.), Die »General Comments« zu den VN-Menschenrechtsverträgen, Baden-Baden 2005. 
In Anbetracht der Tatsache, dass nur wenige Menschenrechte völkerrechtlich absolut gelten, kreisen zudem viele inhaltliche Kontroversen um die Frage, inwieweit und mit welcher Begründung die einzelnen Rechte eingeschränkt werden dürfen. Ein seit jeher kontrovers diskutiertes Beispiel ist die Meinungsfreiheit, die ihre sachlichen Schranken u.U. im Gemeinwohl (öffentliche Ordnung, Sicherheit des Staates, Gesundheitsschutz, Moral und Sittlichkeit etc.) findet und mit den Rechten Dritter kollidieren kann, etwa im Falle von »Rassenhetze« oder Beleidigung..$^{14}$ Aktuell kreist die Kontroverse um das Thema Meinungsfreiheit und religiöse Diffamierung ${ }^{15}$ - ein Thema, das durch den Karikaturenstreit zusätzliche Brisanz gewonnen hat und inzwischen UN-Menschenrechtsorgane beschäftigt. So brachte Pakistan als Wortführer der Organisation Islamischer Konferenz bei der 4. Sitzung des UN-Menschenrechtsrates im März 2007 eine umstrittene Resolution gegen die Diffamierung von Religionen ein, die sogar verabschiedet wurde. ${ }^{16}$ Selbst absolute, notstandsfeste Menschenrechte wie das Folterverbot sind vor Relativierungsversuchen nicht gefeit. ${ }^{17}$

Obwohl die Unteilbarkeit der Menschenrechte unentwegt betont wird, wird zudem den verschiedenen Menschenrechten nicht immer die gleiche Bedeutung beigemessen. Dies verdeutlicht die althergebrachte, regional unterschiedlich geführte Debatte um die Gleichwertigkeit der bürgerlich-politischen und der wirtschaftlichen, sozialen und kulturellen Menschenrechte. Erst seit den 1990er Jahren haben die sog. wsk-Rechte an Bedeutung gewonnen und wird ihre Justiziabilität zusehends anerkannt. ${ }^{18}$ Nach einer langwierigen und mühsamen Kompromisssuche hat die UN-Generalversammlung an 10. Dezember 2008 ein Zusatzprotokoll zum UN-Sozialpakt verabschiedet, das ein entsprechendes internationales Beschwerdeverfahren vorsieht. ${ }^{19}$

Schließlich ist im Detail umstritten, wer durch die Menschenrechte, wem gegenüber auf welche Weise berechtigt bzw. verpflichtet ist. Während die Menschenrechte vornehmlich als Individualrechte ausgestaltet sind, gibt es beispielsweise regional unterschiedlich geführte Kontroversen darüber, inwieweit auch Kollektive (Völker,

14 Vgl. z.B. Rainer Huhle, »Wie weit geht die Meinungsfreiheit? Ein Rückblick aus Anlass des 60-jährigen Bestehens der Allgemeinen Erklärung der Menschenrechte« in: Zeitschrift für Menschenrechte, Jg. 2, Nr. 2 (2008), S. 132-145.

15 Vgl. Eckard Klein, Meinungsäußerungsfreibeit versus Religions- und Glaubensfreibeit, Berlin 2007.

16 Vgl. www2.ohchr.org/english/bodies/hrcouncil/4session/index.htm (abgerufen am 28.12.2008).

17 Zur umfänglichen Folterdiskussion siehe beispielsweise die Themenausgabe von: Aus Politik und Zeitgeschichte, Nr. 36 (2006).

$18 \mathrm{Vgl}$. Kristina Klee, Die progressive Verwirklichung wirtschaftlicher, sozialer und kultureller Menschenrechte, Stuttgart u.a. 2000; Asbjørn Eide et al. (Hg.), Economic, Social and Cultural Rights, Dordrecht u.a. 2001; Bertrand G. Rancharan, Judicial Protection of Economic, Social and Cultural Rights, Leiden u.a. 2005; Scott Leckie / Anne Gallagher, Economic, Social and Cultural Rights. A Legal Ressource Guide, Philadelphia 2006; Thomas Griegerich / Andreas Zimmermann (Hg.), Wirtschaftliche, soziale und kulturelle Rechte im globalen Zeitalter, Berlin 2008; Varun Gauri / Daniel M. Brinks (Hg.), Courting Social Justice. Judicial Enforcement of Social and Economic Rights in the Developing World, Cambridge 2008.

19 Vgl. Resolution A/RES/63/117. 
Minderheiten) menschenrechtliche Ansprüche erheben können. Auch wird gefordert, über den Staat hinaus internationale Organisationen wie die Weltbank oder die Welthandelsorganisation sowie nicht-staatliche Akteure, allen voran Wirtschaftsunternehmen, auf die Achtung der Menschenrechte zu verpflichten..$^{20}$ Umstritten ist weiterhin, inwieweit die Staaten außerhalb ihres jeweiligen Staatsgebietes sog. extraterritoriale Verpflichtungen innehaben. ${ }^{21}$

$\mathrm{Da}$ die rechtliche Festschreibung und Ausdeutung der Menschenrechte und ihres Geltungsbereichs nicht ein für allemal abgeschlossen ist, sondern einem historischen Wandel unterliegt und beachtliche kulturelle Varianzen aufweist, ist für das Projekt einer rechtlichen Universalisierung der Menschenrechte wichtig, dass beständig der Versuch unternommen wird, über eine offene und kritische Auseinandersetzung um die Interpretation bestehender (oder auch die Festschreibung neuer) Menschenrechte und ihres Geltungsbereiches ein gemeinsames Grundverständnis herzustellen, das den Gegebenheiten und Problemen der jeweiligen Zeit und des jeweiligen Ortes Rechnung trägt. In diesem Sinne erarbeiten beispielsweise die Menschenrechtsvertragsorgane Interpretationsvorgaben für die Länderberichte der Vertragsstaaten. ${ }^{22}$

\subsection{Institutionelle Schwächen des UN-Menschenrechtsschutzes}

Die internationale Staatengemeinschaft erwies sich bisher nicht als willens oder fähig, den universellen Menschenrechtsschutz mit effektiven Durchsetzungsmechanismen auszustatten, wie es eines der ursprünglichen Ziele der 1946 eingesetzten UNMenschenrechtskommission war. Trotz wichtiger institutioneller Reformen - wie der Errichtung eines Hochkommissars für Menschenrechte (1993), eines Internationalen Strafgerichtshofes (1998/2002) oder mit Vorbehalt auch des neuen Menschenrechtsrates (2006), der sich erst noch bewähren muss - blieb bezeichnenderweise die Vision eines Weltgerichtshofes für Menschenrechte bislang bar jeglicher Realisierungschance. ${ }^{23}$

Viele Staaten unterwerfen sich nicht einmal den bestehenden schwachen Überwachungsinstrumenten der grundlegenden UN-Menschenrechtsverträge. Bereits den Berichtspflichten, die diese Abkommen vorsehen, kommen einige Regierungen nicht oder nur mit erheblicher Verspätung nach. Eine Staatenbeschwerde ist zwar bei vie-

20 Vgl. Michael Windfuhr (Hg.), Beyond the Nation State. Human Rights in Times of Globalization, Uppsala 2005; Andrew Clapham, Human Rights Obligations of Non-State Actors, Oxford 2006; Holger Hestermeyer, Human Rights and the WTO, Oxford 2007; Katharina Spieß, »Wirtschaft und Menschenrechte« in: Daniela Hinze (Hg.), Für Freibeit und Menschenwürde. 60 Jahre Allgemeine Erklärung der Menschenrechte, Berlin 2008, S. 84-107.

21 Vgl. Fons Coomans / Menno T. Kaminga (Hg.), Extraterritorial Application of Human Rights Treaties, Antwerpen 2004; Sigrun Skogly, Beyond National Borders: States Human Rights Obligations in International Cooperation, Antwerpen 2006.

22 Vgl. Deutsches Institut für Menschenrechte, Die »General Comments", aaO. (Fn. 13).

23 Die Idee wurde ursprünglich von Australien vorgetragen (z.B. UN Doc. E/1371 vom 23.6.1949) und in jüngster Zeit von Manfred Nowak wieder ausgegriffen; vgl. Manfred Nowak, "Ein Weltgerichtshof für Menschenrechte. Eine utopische Forderung?« in: Vereinte Nationen, Nr. 5 (2008), S. 205-211. 
len Menschenrechtsabkommen möglich, wurde aber auf UN-Ebene bislang nie genutzt. Das wichtigere Instrument der Individualbeschwerde kann nur bei einer $\mathrm{Zu}$ stimmungserklärung durch den beschuldigten Staat (IECRD, CAT, ICRMW) oder bei der Ratifikation eines Zusatzprotokolles (ICCPR, CEDAW, ICRPD) zur Anwendung kommen, die lediglich bei 111 (ICCPR), 96 (CEDAW) bzw. 27 (ICRPD) Vertragsparteien vorliegt (Stand: Dezember 2008). Bei der Kinderrechtskonvention ist eine Individualbeschwerde (noch) nicht vorgesehen. Im Falle des ICESCR wurde ein entsprechendes Zusatzprotokoll erst 2008 verabschiedet und zur Ratifikation ausgelegt. Eigenständige Untersuchungen bei schwerwiegenden oder systematischen Menschenrechtsverletzungen durch Staaten können die jeweiligen Kontrollausschüsse zudem lediglich bei drei der o.g. Abkommen einleiten, und zwar nur bei fehlendem Vorbehalt des beschuldigten Staates (CAT, CEDAW) oder bei Ratifikation eines Zusatzprotokolls, wie neuerdings zum ICESCR.

Angesichts der Schwäche der internationalen Durchsetzungsinstrumente sagt die reine Ratifikation universeller Menschenrechtsabkommen daher nur bedingt etwas über den Willen der Staaten aus, die Menschenrechte zu achten und umzusetzen. Im Sinne eines rationalen Kosten-Nutzen-Kalküls kann es selbst normverletzenden Regimen zupass kommen, wenn sie prestigeträchtige Menschen-rechtsabkommen ratifizieren, da sich daraus oft keine weit reichenden Handlungskonsequenzen ergeben. ${ }^{24}$ Dies gilt gerade dann, wenn auch das regionale commitment zu den Menschenrechten gering ist und nur schwache nationale Durchsetzungsinstrumente des Menschenrechtsschutzes bestehen. ${ }^{25}$

\subsection{Der regionale Menschenrechtsschutz - kein einheitliches Bild}

Durch die Mitgliedschaft in regionalen Organisationen, wie der EU, dem Europarat, der OAS oder der OAU, sowie durch die Ratifikation regionaler Menschenrechtsabkommen gehen viele Staaten zusätzliche menschenrechtliche Verpflichtungen ein, die teils einer politischen, teils einer rechtlichen Prüfung unterzogen werden (können). Die regionalen Menschenrechtsabkommen ${ }^{26}$ zeigen, dass bei Aufrechterhaltung der völkerrechtlich verbürgten Menschenrechtsstandards auf regionaler Ebene durchaus Raum für speziellere Normierungen besteht. Vor dem Hintergrund regional unterschiedlich gelagerter Problemlagen kann dies ausgesprochen sinnvoll sein. Problematisch wird es allerdings dann, wenn regionale Rechtsnormierungen und -auslegungen die universellen Standards aushöhlen. Dies ist beispielsweise bei einigen Formulierungen und Interpretationen der Allgemeinen Erklärung

24 Vgl. Emilie M. Hafner-Burton / Kiyoteru Tsutsui, »Justice Lost! The Failure of International Human Rights Law to Matter Where Needed Most « in: Journal of Peace Research, Vol. 44, Nr. 4 (2007), S. 407-425.

25 Vgl. Oona A. Hathaway, »Why Do Countries Commit to Human Rights Treaties « in: Journal of Conflict Resolution, Vol. 51, Nr. 4 (2007), S. 588-621.

26 Vgl. Waldemar Hummer / Wolfram Karl (Hg.), Regionaler Menschenrechtsschutz, Dokumente und Einführungen, Bd. 1: Allgemeiner Schutzbereich (zwei Teilbände), Baden-Baden 2009. 
der Menschenrechte im Islam von 1980 oder auch die Kairoer Erklärung über Menschenrechte im Islam von 1990 der Fall. Dabei handelt es sich um zwei rechtlich unverbindliche Dokumente, die den Menschenrechtsbezug ausschließlich in den Dienst einer Glaubenslehre stellen und somit dem Universalitätsgedanken zuwiderlaufen. $^{27}$

Sofern aber der Universalitätsanspruch nicht aufgegeben wird, kann der weltweite Ausbau des regionalen Menschenrechtsschutzes die rechtliche Universalisierung der Menschenrechte erheblich befördern. Bislang ist jedoch der regionale Menschenrechtsschutz nur in Europa ${ }^{28}$, Amerika ${ }^{29}$ und Afrika ${ }^{30}$ mit entsprechenden Institutionen und Abkommen ausgestaltet - und differiert erheblich hinsichtlich seiner Durchsetzungskraft und praktischen Bedeutung. Am stärksten entwickelt ist er in Europa, wo vor allem der Europäische Gerichtshof für Menschenrechte - bei aller Reformbedürftigkeit - ein wichtiges Instrument der Rechtsdurchsetzung darstellt. Trotz fehlender Vollstreckungsgewalt werden dessen Urteile zu 97\% umgesetzt, wenn auch oft mit zeitlicher Verzögerung. ${ }^{31}$

\subsection{Die große Bedeutung des nationalen Menschenrechtsschutzes}

Da der internationale Menschenrechtsschutz subsidiär ausgestaltet ist, erlangt der nationale Schutz der Menschenrechte herausgehobene Bedeutung. Erst wenn dieser versagt, kommt der regionale oder universelle Menschenrechtsschutz zum Tragen. Für die weltweite rechtliche Geltung internationaler Menschenrechtsnormen ist daher die Frage bedeutsam, inwieweit die Menschenrechte im nationalen Recht verankert sind und ihre Umsetzung innerhalb der Staaten institutionell abgesichert ist.

Grundsätzlich steht es den Staaten dabei frei, ob sie internationalen Menschenrechtsverträgen im nationalen Recht automatisch Geltung zusprechen oder deren Inhalt auf dem Weg der Gesetzgebung, etwa mittels inhaltlich identischer Gesetze

27 Vgl. Eibe Riedel, Der internationale Menschenrechtsschutz, aaO. (Fn. 8), S. 40. Siehe auch Anna Würth, Dialog mit dem Islam als Konfliktprävention. Zur Menschenrechtspolitik gegenüber islamisch geprägten Staaten, Berlin 2003.

28 Vgl. z.B. Jens-Meyer Ladewig, Europäische Menschenrechtskonvention - EMRK: Handkommentar, Baden-Baden 2006; Sebastian Heselhaus / Carsten Nowak, Handbuch der Europäischen Grundrechte, München u.a. 2006; Klaus Brummer, Der Europarat: Eine Einführung, Wiesbaden 2008.

29 Vgl. u.a. David Harris / Stephen Livingstone (Hg.), The Inter-American System of Human Rights, Oxford 1998; Wolfgang S. Heinz, »Der Interamerikanische Menschenrechtsschutz « in: Nicole Janz / Thomas Risse (Hg.), Menschenrechte - Globale Dimensionen eines universellen Anspruchs, Baden-Baden 2007, S. 81-99.

30 Vgl. z.B. Vincent O. Orl Nmehielle, The African Human Rights System: Its Laws, Practices and Institutions, The Hague 2001; Obiora Chinedu Okafor, The African Human Rights System. Activist Forces and International Institutions, Cambridge 2007; Malcolm Evans / Rachel Murray, The African Charter on Human and Peoples' Rights: The System in Practice 1986-2006, Cambridge 2008.

31 Vgl. Anne Peters, Einführung in die Europäische Menschenrechtskonvention, München 2003, S. 253. 
oder entsprechender Zustimmungsgesetze, in nationales Recht transformieren. Auch verlangt das Völkerrecht von den Staaten nicht zwingend, den einzelnen Menschen das Recht einzuräumen, sich vor innerstaatlichen Organen direkt auf die in Menschenrechtsabkommen verankerten Rechte zu berufen. Es reicht, wenn analoge Verfassungsgarantien oder Gesetzesbestimmungen geltend gemacht werden können. ${ }^{32}$ In diesem Sinne ist jeweils zu prüfen, ob das nationale Recht menschenrechtskonform ausgestaltet ist und ob es entsprechende Rechtsgarantien beinhaltet. Diesbezüglich lassen sich erhebliche Länderunterschiede zwischen, aber auch innerhalb der Weltregionen feststellen. ${ }^{33}$

Es ist nun hier nicht der Raum, um unter menschenrechtlichen Gesichtspunkten einen Vergleich nationaler Rechtsordnungen zu etablieren und zu prüfen, inwieweit sich ggf. die Partikularität einzelstaatlicher Regelungen mit dem egalitären und universellen Anspruch der Menschenrechte reibt. Einige grundlegende Bemerkungen seien jedoch erlaubt: Die Grundrechtskataloge nationaler Verfassungen, die teilweise vor der Entwicklung des internationalen Menschenrechtssystems entstanden sind, umfassen oft nicht alle Menschenrechte, die in der Allgemeinen Erklärung der Menschenrechte und den beiden grundlegenden UN-Pakten verankert sind. Während beispielsweise einige, meist jüngere Verfassungen (z.B. in Lateinamerika oder auch Südafrika) wirtschaftliche, soziale und kulturelle Rechte in ihre Grundrechtskataloge aufgenommen haben, beschränken sich andere (wie Deutschland) weitgehend auf bürgerliche und politische Rechte. Hinzu kommt, dass die nationalstaatlich formulierten Grundrechte nicht immer als Menschenrechte ausgestaltet sind, die jedem Menschen zustehen; teilweise haben sie »nur « den Status von Bürgerrechten inne, die den jeweiligen Staatsangehörigen zukommen.

Fehlt der spezielle Grundrechtsschutz, ist daher zu prüfen, ob weitere Rechtsnormen und Institutionen die Menschenrechte garantieren und schützen. So sind in Deutschland etwa Staatsangehörigkeitsvorbehalte gegenüber EU-Ausländern im Anwendungsbereich des Gemeinschaftsrechts unzulässig, und das Grundrecht der allgemeinen Handlungsfreiheit schützt u.a. die Versammlungs- und Berufsfreiheit auch von Nicht-EU-Ausländern, obwohl die entsprechenden Grundrechte laut Grundgesetz nur Deutschen garantiert sind. ${ }^{34}$ Aufgrund des Sozialstaatsprinzips und des entwickelten Arbeits- und Sozialrechts sind in Deutschland zudem wirtschaftliche und soziale Menschenrechte rechtlich geschützt, obwohl diese nicht als Grundrechte im Grundgesetz verankert sind.

Wichtig ist aber vor allem, dass das nationale Recht nicht nur formal besteht, sondern auch rechtspraktische Bedeutung entfaltet. Hierfür ist ein Mindestmaß an Rechtsstaatlichkeit vonnöten, einschließlich einer effektiven unabhängigen Justiz. Autoritäre Willkürherrschaft ist damit nicht vereinbar. Umgekehrt ist selbst ein mi-

32 Vgl. Walter Kälin / Jörg Künzli, Universeller Menschenrechtsschutz, aaO. (Fn. 9), S.179 f.

33 Vgl. etwa für Lateinamerika: José F. Palomino Manchego / José Carlos Remotti Carbonell (Hg.), Derechos Humanos y Constitución en Iberoamérica, Lima 2002.

34 Vgl. einführend: Bodo Pieroth / Bernhard Schlink, Grundrechte. Staatsrecht II, Heidelberg 2007, S. $28 \mathrm{ff}$. 
nimales liberal-repräsentatives Demokratieverständnis hinsichtlich der Menschenrechte äußerst anspruchsvoll. ${ }^{35}$ Der definitorische Zusammenhang zwischen Demokratie, Rechtsstaatlichkeit und Menschenrechten erweist sich als eng. Letztlich ist daher immer zu prüfen, inwieweit das Verfassungsrecht im Rahmen einer demokratischen, rechtsstaatlichen Verfassungspraxis zum Tragen kommt (siehe Kapitel 3).

\subsection{Menschenrechtsförderung in rechtlich-institutioneller Hinsicht}

Aus rechtlicher Perspektive erfordert die Universalisierung der Menschenrechte, dass diese universell kodifiziert sind, sich möglichst alle souveränen Staaten zur Einhaltung und Umsetzung verpflichten, dass zumindest ein inhaltliches Grundverständnis der Rechte vorliegt und effektive Instrumente zur Rechtsdurchsetzung bestehen. Der zugrunde liegende Handlungsmodus ist dabei regulativ und regelgeleitet, setzt also auf die Normierung, Anerkennung und Durchsetzung von Rechtsnormen. Bezeichnenderweise zielen viele menschenrechtspolitische Maßnahmen auf die Etablierung und Stärkung menschenrechtlicher Normen und Institutionen ab.

Dies belegt beispielsweise der jüngste Menschenrechtsbericht der Bundesregierung. ${ }^{36}$ Diese bemüht sich in ihrer bilateralen und multilateralen Menschenrechtspolitik u.a. um die Ächtung der Todesstrafe, wirbt in Drittstaaten für den Beitritt zur UN-Antifolterkonvention und begrüßt das - noch nicht in Kraft getretene - Internationale Übereinkommen zum Schutz aller Personen vor dem Verschwindenlassen. Weiterhin setzt sich die Bundesregierung auf UN-Ebene etwa für eine Stärkung des Menschenrechtsrates, der Sonderberichterstatter, des Hochkommissariats für Menschenrechte und der Menschenrechtsvertragsorgane ein, unterstützt zudem den Internationalen Strafgerichtshof und beteiligt sich am Reformprozess des Europäischen Gerichtshofes für Menschenrechte. Nach zeitweiliger Skepsis hat sie sogar die Verabschiedung des Zusatzprotokolls zum UN-Sozialpakt mitgetragen und sich im Menschenrechtsaktionsplan verpflichtet, das Protokoll bald zu zeichnen und den Ratifikationsprozess voranzutreiben. Das Zusatzprotokoll zur UN-Antifolterkonvention, das präventive Besuchsmechanismen vorsieht, und das UN-Übereinkommen für die Rechte von Menschen mit Behinderungen wurden inzwischen gezeichnet bzw. ratifiziert. Allerdings steht seit Jahren die Ratifikation der revidierten Europäischen Sozialcharta aus und wurde der deutsche Vorbehalt zur UN-Kinderrechtskonvention noch nicht zurückgenommen.

35 Vgl. Michael Krennerich, »Demokratie und Menschenrecht. Wie eng gehören beide zusammen?« in: böll Thema, Nr. 3 (2008), S. 31 f.; Petra Bendel et al. (Hg.), Zwischen Demokratie und Diktatur, Opladen 2002.

36 Vgl. Deutscher Bundestag, Unterrichtung durch die Bundesregierung: Achter Bericht der Bundesregierung über ibre Menschenrechtspolitik in den auswärtigen Beziebungen und in anderen Politikbereichen, BT-Drs. 16/10037, 16.7.2008. In kritischer Würdigung: Michael Krennerich, »Menschenrechte als Querschnittsaufgabe. Ein Streifzug durch den jüngsten Menschenrechtsbericht der Bundesregierung « in: Zeitschrift für Menschenrechte, Jg. 2, Nr. 2 (2008), S. 150-158. 
Aktiv lobbyiert werden die deutsche wie auch andere Regierungen durch nichtstaatliche Menschenrechtsorganisationen, die eine treibende Kraft für die Fortentwicklung des internationalen Menschenrechtsschutzes darstellen, auch bei der Normsetzung. Viele internationale und regionale Vertragswerke wurden erst durch lang anhaltende Kampagnen und die Eingaben nicht-staatlicher Organisationen initiiert und ausgestaltet. ${ }^{37}$ Prominente Beispiele sind hier die UN-Anti-Folterkonvention oder auch die Normierung von Menschenrechten zum besonderen Schutz von Frauen und Kindern. Auch die wachsende Bedeutung des internationalen Schutzes der wirtschaftlichen, sozialen und kulturellen Menschenrechte geht maßgeblich auf NGOs zurück. ${ }^{38}$

Nicht minder wichtig sind der Ausbau und die Förderung des nationalen Menschenrechtsschutzes. Allgemein ist hier die Entwicklung eines demokratischen Rechtsstaates förderlich. Im Speziellen können menschenrechtsbezogene Gesetzesreformen oder die Errichtung und Stärkung entsprechender Institutionen wichtig sein (z.B. Ombudspersonen, Wahrheitskommissionen, Menschenrechtsbeauftragte und -institute, parlamentarische Kontrollausschüsse oder eine unabhängige Verfassungsgerichtsbarkeit). ${ }^{39}$ In diesem Sinn geht beispielsweise die Menschenrechtsförderung in der staatlichen Entwicklungszusammenarbeit Deutschlands eng mit der Förderung von Demokratie und Rechtsstaatlichkeit einher und zielt auch auf die Stärke menschenrechtsrelevanter Institutionen ab. ${ }^{40}$ Auch inter- oder supranationale Organisationen drängen - wie etwa die EU gegenüber der Türkei ${ }^{41}$ - auf eine men-

37 Vgl. Andrea Liese, »Menschenrechtsschutz durch Nichtregierungsorganisationen « in: Aus Politik und Zeitgeschichte, B 46-47 (1998), S. 36-42; Nils Geißler, »Einfluss und Rolle der Nichtregierungsorganisationen beim Schutz der Menschenrechte in: Erwin Müller/ Patricia Schneider / Kristina Thony (Hg.), Menschenrechtsschutz, Baden-Baden 2002, S. 62-78; Claire Breen, »The Role of NGOs in the Formulation of and Compliance with the Optional Protocol to the Convention on the Rights of the Child on Involvement of Children in Armed Conflict « in: Human Rights Quarterly, Vol. 25, Nr. 2 (2003), S. 453-481; Barbara Lochbihler, »Die Rolle von Nichtregierungsorganisationen im internationalen Menschenrechtsschutz. Das Beispiel von Amnesty International« in: Sven Bernhard Gareis / Gunter Geiger (Hg.), Internationaler Schutz der Menschenrechte. Stand und Perspektiven im 21. Jabrbundert, Opladen/Farmington Hills, S. 125-139.

38 Vgl. Michael Windfuhr, "Die Bedeutung der sozialen Menschenrechte in der Arbeit internationaler Menschenrechtsorganisationen « in: Ulla Selchow / Franz-Josef Hutter (Hg.), Menschenrechte und Entwicklungszusammenarbeit, Wiesbaden 2004, S. 195-207; Michael Krennerich, »Die Förderung sozialer Menschenrechte durch nicht-staatliche Organisationen « in: MenschenRechtsMagazin, Jg. 10, H. 1 (2005), S. 30-48.

39 Vgl. etwa: Kamal Hossain et al. (Hg.): Human Rights Commissions and Ombudsman Offices. National Experiences throughout the World, The Hague u.a. 2000; Valentin Aichele, Nationale Menschenrechtsinstitutionen. Ein Beitrag zu nationalen Implementierung von Menschenrechten, Frankfurt a.M. u.a. 2003; Comisión Andina de Juristas et al., Defensorias del pueblo en la Región Andina: experiencias comparadas, Lima 2001; Anna-Elina Pohjolainen, The Evolution of National Human Rights Institutions, Copenhagen 2006.

40 Vgl. Michael Krennerich, »Entwicklung und Menschenrechte« in: Hinze, Für Freibeit und Menschenwürde, aaO. (Fn. 20), S. 48-82. 
schenrechtskonforme Ausgestaltung der nationalen Rechtsordnungen. Vor allem aber fordern nationale und internationale NGOs immer wieder nationale Reformen ein oder stoßen diese selbst an. Ein Beispiel hierfür ist die Menschenrechtsorganisation FIAN, die sich nicht nur international, sondern auch in den jeweiligen Ländern dafür einsetzt, dass das Recht auf Nahrung rechtlich verankert und institutionell geschützt wird. ${ }^{42}$

\section{Menschenrechte in der politischen Praxis}

Der Staat ist der vornehmliche Adressat menschenrechtlicher Ansprüche, und es bedarf politischer Entscheidungsprozesse, um aus theoretisch begründeten Menschenrechten auch positive Rechte zu machen und diese dann umzusetzen. Dieser Umstand erfordert es, die politische Praxis innerhalb und zwischen den Staaten in Augenschein zu nehmen.

\subsection{Die Lage der Menschenrechte - ein ernüchterndes Bild}

Eine Vielzahl nationaler wie internationaler, staatlicher wie nicht-staatlicher Organisationen und Institutionen betreiben ein mehr oder minder professionelles Menschenrechts-Monitoring. Dazu gehören vor allem nationale, regionale und internationale NGOs ${ }^{43}$, weiterhin: Menschenrechtsinstitute, Ombudspersonen oder Wahrheitskommissionen sowie die zahlreichen Kontrollorgane des universellen und regionalen Menschenrechtsschutzes, etwa auf der Ebene der Vereinten Nationen, der ILO, des Europarates etc. Auch die nationale Justiz und internationale Strafgerichte tragen erheblich zur Aufklärung bei, ebenso die Medien.

Art, Systematik und Umfang des Monitorings unterscheiden sich allerdings erheblich, u.a. abhängig davon, was untersucht wird: Geht es um die Menschenrechtsperformanz der Regierung oder allgemein um die Menschenrechtslage in einem Land? Stehen die Rechte der gesamten Bevölkerung im Blickpunkt oder bestimmte Zielgruppen und Landesteile? Werden nur die bürgerlichen und politischen oder auch die wirtschaftlichen, sozialen und kulturellen Rechte betrachtet? Werden lediglich Menschenrechtsverletzungen oder auch positive Maßnahmen des Schutzes und der Förderung der Menschenrechte erfasst? Ist das Monitoring eher ereignisbezogen oder eher indikatorengestützt? Die technischen und methodischen Unterschiede bei der Erhebung und Dokumentation der Daten sind erheblich. Dennoch ergibt sich aus der Gesamtschau der verfügbaren Berichte in der Regel ein

41 Vgl. die entsprechenden Fortschrittsberichte der Europäischen Kommission seit 1998 (abrufbar unter: http://ec.europa.eu/enlargment/press_corner/key-documents/reports_ nov_2008_de.htm) (Stand: 28.12.2008).

42 Vgl. www.fian.org und www.fian.de.

43 Für NGOs stehen hierbei methodische Hilfen zur Verfügung, etwa seitens des 1982 gegründeten Netzwerks HURIDOCS (Human Rights Information and Documentation System International): www.huridocs.org/tools (Stand: 28.12.2008). 
fundiertes und differenziertes Bild der menschenrechtlichen Performanz der Regierung oder der Menschenrechtslage in den jeweiligen Ländern, so dass die menschenrechtliche Weltkarte kaum mehr »blinde Flecken« aufweist.

Für sozialwissenschaftliche Zwecke werden die entsprechenden Informationen meist nochmals eigens aufbereitet. ${ }^{44}$ Gewisser Beliebtheit erfreuen sich hierbei Indizes, mittels derer qualitative wie quantitative Informationen kodiert werden. Auf Grundlage der »Country Reports on Human Rights Practices « des U.S. State Department sowie ergänzend auch der Jahresberichte von Amnesty International erstellt beispielsweise der CIRI-Index für die Jahre seit 1981 standardisierte, quantitative Informationen darüber, inwieweit Regierungen internationale Menschenrechte achten - und erfasst dabei folgende Rechte bzw. Rechtsverletzungen: willkürliche Tötungen, Folter, »Verschwindenlassen « und politische Haft (einzeln und zusammengefasst im »Physical Integrity Rights Index«), weiterhin: freie Meinungsäußerung, Versammlungs- und Vereinigungsfreiheit, Freizügigkeit, Religionsfreiheit, politische Mitwirkung bei Wahl der Regierung und Arbeitnehmerrechte (einzeln und zusammengefasst im »Empowerment Rights Index«) sowie politische, wirtschaftliche und soziale Rechte von Frauen und die Unabhängigkeit der Justiz.45 Anna Würth zufolge ${ }^{46}$ ist der CIRI-Index wohl der umfassendeste Menschenrechtsindex - und doch misst er vornehmlich, wenngleich nicht ausschließlich, die Einhaltung der bürgerlich-politischen Rechte.

Andere Indizes beschränken sich gänzlich auf bürgerliche und politische Rechte. Hier ist beispielsweise die seit den frühen 1980er Jahren bestehende »Political Terror Scale« zu nennen, der für alle Staaten weltweit seit 1976 den Grad des »Staatesterrors « anhand irregulärer staatlichen Tötungen, Folter, »Verschwindenlassen « und politischer Inhaftierungen bemisst (also die Rechte des »Physical Integrity Rights Index « beim CIRI-Index). Auch hier werden die entsprechenden Informationen den Länderberichten von Amnesty International und des U.S. State Department entnommen, kodiert und quantitativ darstellt. ${ }^{47}$ Dabei wird ein Länder-Ranking erstellt, auf das Amnesty International bewusst verzichtet. Zu den »worst offenders « im Zeitraum von 1996 und 2006 gehören demnach Irak, Kolumbien, Sudan, die Demokratische Republik Kongo, Afghanistan, Burundi, Algerien, Nordkorea, Myanmar, Angola und China.

Eindeutig beschränkt auf die bürgerlich-politischen Rechte bleibt auch der viel zitierte, seit 1972 jährlich erhobene »Freedom House Index«. Er bestimmt für die jeweiligen Länder allerdings nicht die Performanz der Regierungen, sondern den

44 Vgl. einführend: Todd Landman, Studying Human Rights, London/New York 2006. Zu den an Bedeutung gewinnenden statistischen Methoden siehe: Jana Asher / David Banks / Fritz J. Scheuren (Hg.), Statistical Methods for Human Rights, New York 2008.

45 Vgl. David L. Cingranelli / David L. Richards, The Cingranelli-Richards (CIRI) Human Rights Data Project Coding Manual Version 2008.3.13 (http://ciri.binghamton.edu/documentation/ciri_coding_guide.pdf) (zuletzt abgerufen: 28.12.2008).

46 Vgl. Anna Würth, »Monitoring and Measuring Human Rights - A Brief Survey« in: Die Friedens-Warte, Jg. 81, Nr. 1 (2006), S. 71-86.

47 Vgl. http://www.politicalterrorscale.org (zuletzt abgerufen: 28.12.2008). 
»Freiheitsgrad « aus Sicht der Individuen, der sowohl von staatlichen als auch von nicht-staatlichen Akteuren beeinflusst wird. Die Quellengrundlage des Indexes sind insbesondere Medienberichte und Informationen unabhängiger Organisationen (gerade auch aus dem Bereich der Menschenrechte), die von Länderanalytikern anhand einer zehn politischen Rechte und 15 bürgerlichen Freiheitsrechte umfassenden Checkliste ausgewertet werden. Die entsprechenden Informationen werden in eine Skala übergeführt und bestimmen den jeweiligen Status der 193 Länder und 15 abhängigen Gebiete als »free«, »partly free« und »non free «. ${ }^{88}$ Für den Berichtszeitraum 2007 wurden insgesamt 90 der 193 Staaten als »frei« eingestuft. Die »Weltkarte der Freiheit « weist hierbei den amerikanischen Kontinent, Europa sowie Australien und Ozeanien mit einigen Ausnahmen als weitgehend »frei« aus, Afrika und Asien hingegen als überwiegend »halbfrei« und »nicht-frei«. Die am schlechtesten bewerteten Staaten sind mit jeweils gleichen Index-Werten Myanmar, Kuba, Libyen, Nordkorea, Somalia, Sudan, Turkmenistan und Usbekistan.

Die wirtschaftlichen, sozialen und kulturellen Menschenrechte werden, obwohl es inzwischen ein entsprechendes Monitoring gibt, von den gängigen Menschenrechtsindizes kaum erfasst. Demgemäß wird ein umfassender, beide »Generationen " von Rechten einschließender, quantitativer Menschenrechtsindex gefordert. ${ }^{49}$ Mitunter werden die wsk-Rechte sogar in qualitativen Menschenrechtsberichten vernachlässigt. So nehmen beispielsweise die o.g. Länderberichte des US State Department - über Arbeitnehmerrechte hinaus - nur am Rande auf Verletzungen der wsk-Rechte Bezug. Auch im Länderteil des jüngsten Menschenrechtsberichts der deutschen Bundesregierung finden Verletzungen der wsk-Rechte kaum Erwähnung - trotz des nachdrücklichen Bekenntnisses zur Förderung dieser Rechte in der internationalen Zusammenarbeit Deutschlands. ${ }^{50}$ Dies hat maßgeblich damit zu tun, dass die wsk-Rechte vornehmlich als (teure) Teilhabe- oder Leistungsrechte wahrgenommen werden, ihr Abwehr- und Schutzcharakter aber tendenziell vernachlässigt wird. ${ }^{51}$ Bezeichnenderweise wird in Bezug auf die wirtschaftlichen, sozialen und kulturellen Menschenrechte oft auf Makroindikatoren hingewiesen, die beispielsweise in den »Physical Quality of Life Index « oder den »Human Development Index « des UN-Entwicklungsprogramms einfließen. ${ }^{52}$ Diese sagen zwar möglicherweise etwas über das - von vielfältigen Faktoren abhängige - Ergebnis einer

48 Vgl. http://www.freedomhouse.org (zuletzt abgerufen: 28.12.2008).

49 Vgl. etwa Sean Holland, Ranking Rights: Problems and Prospects for a Quantitative Global Human Rights Index, in: Measurement \& Human Rights Issue Paper, Vol. 1, Issue 4 (2008).

50 Vgl. Deutscher Bundestag, Unterrichtung durch die Bundesregierung, aaO. (Fn. 36).

51 Vgl. Michael Krennerich, »Soziale Menschenrechte sind Freiheitsrechte! Plädoyer für ein freiheitliches Verständnis wirtschaftlicher, sozialer und kultureller Rechte« in: Deutsches Institut für Menschenrechte et al. (Hg.), Jahrbuch Menschenrechte 2007, Frankfurt a.M. 2006, S. 57-66.

52 Vgl. David L. Cingranelli / David L. Richards, Measuring Government Respect for Economic Human Rights, Paper prepared for the 2004 Annual Meeting of the Midwest Polical Science Association, Chicago, IL, 2004. 
menschenrechtsorientierten Entwicklung aus, wenig aber über die Regierungspraktiken in Bezug auf die wsk-Rechte.

Hierzu sind direkt die Berichte von $\mathrm{NGOs}^{53}$ oder auch von $\mathrm{UN}-\mathrm{Organen}^{54} \mathrm{zu}$ konsultieren. Sie lassen erhebliche Unterschiede zwischen den Ländern erkennen, belegen aber, dass die wsk-Rechte trotz einiger Fortschritte weltweit verletzt oder zumindest nicht hinreichend umgesetzt werden. Auch zeigen sie, dass die Verletzungen der wsk-Rechte und der bürgerlich-politischen Rechte oft Hand in Hand gehen. Ein eindrückliches Beispiel für das Zusammenwirken von Menschenrechtsverletzungen ist Zimbabwe, wo in den vergangenen Jahren die Verfolgung der gesellschaftspolitischen Opposition mit groß angelegten Landvertreibungen, der Zerstörung städtischer Armenviertel und einer politisch verursachten Ernährungs- und Gesundheitskrise einherging. ${ }^{55}$

Insgesamt zeigt die politische Praxis, dass die Menschenrechte in Teilen der Welt systematisch und andauernd verletzt werden. Mit Blick auf den Prozess der Universalisierung der Menschenrechte stellt sich dabei die Frage, ob sich die Lage der Menschenrechte zumindest verbessert hat. Für die politischen Rechte und bürgerlichen Freiheiten liegen hier mit dem Freedom-House-Index Zeitreihen vor, die diese Annahme zu bestätigen scheinen: Der prozentuale Anteil der »freien Staaten « weltweit stieg nahezu kontinuierlich von 29\% (1972) auf 47\% (2007) an, während sich im gleichen Zeitraum der prozentuale Anteil der »nicht-freien Staaten« halbierte (vgl. Tab. 2). Dies ist nicht zuletzt auf die weltweiten Liberalisierungs- und Demokratisierungsprozesse der 1980er und 1990er Jahre zurückzuführen, die in dem »Freedom House Index« beachtlich zu Buche schlagen.

53 Vgl. etwa die Berichte des Centre on Housing Rights and Evictions (www.cohre.org), von FIAN International (www.fian.org), der Fédération internationale des ligues de droits de l'Homme (www.fidh.org) oder auch von Amnesty International (www.amnesty.de) und Human Rights Watch (www.hrw.org), die ebenfalls seit einigen Jahren Verletzungen von wsk-Rechten dokumentieren. Siehe auch das Netzwerk ESCR-Net (www.escr-net.org).

54 Vgl. etwa die Abschließenden Kommentare des UN-Ausschusses für wirtschaftliche, soziale und kulturelle Rechte zu den jeweiligen Staatenberichten oder auch die Berichte der UN-Sonderberichterstatter zu einzelnen wsk-Rechten (Ernährung, Gesundheit, Wohnen, Bildung etc.) (www.ohchr.org).

55 Vgl. Inga Morgenstern, »Häuserzerstörung und Vertreibung in Zimbabwe« in: Deutsches Institut für Menschenrechte et al., Jahrbuch Menschenrechte 2007, aaO. (Fn. 50), S. 175-182, sowie aktuelle Zimbabwe-Informationen von Amnesty International (www.amnesty.org). 
Tabelle 2: Länderstatus nach Freedom House (ausgewäblte Jabre)

\begin{tabular}{|l|l|l|l|l|l|l|l|}
\hline Jahr & Länder & \multicolumn{2}{|l|}{ »Free countries « } & \multicolumn{2}{l|}{$\begin{array}{l}\text { »Partly Free } \\
\text { countries }\end{array}$} & \multicolumn{2}{l|}{$\begin{array}{l}\text { Not Free } \\
\text { countries }\end{array}$} \\
\hline & & Anzahl & $\%$ & Anzahl & $\%$ & Anzahl & $\%$ \\
\hline 1972 & 151 & 44 & 29 & 38 & 25 & 69 & 46 \\
\hline 1980 & 162 & 51 & 31 & 54 & 33 & 60 & 37 \\
\hline 1990 & 165 & 65 & 40 & 50 & 30 & 50 & 30 \\
\hline 2000 & 192 & 86 & 45 & 58 & 30 & 48 & 25 \\
\hline 2007 & 193 & 90 & 47 & 60 & 31 & 43 & 22 \\
\hline
\end{tabular}

Quelle: www.freedomhouse.org (abgerufen am 30.12.2008).

Für die »Physical Integrity Rights « sieht der Befund nicht so positiv aus. So lassen die durchschnittlichen »Political Terror Scores « der Nicht-OECD-Staaten zwischen 1976 und 2006 trotz Varianzen im Zeitverlauf insgesamt keine Verbesserungen erkennen. ${ }^{56}$ Bedenklich stimmt darüber hinaus der Umstand, dass selbst etablierte Demokratien wie die USA im Rahmen der Terrorismusbekämpfung der vergangenen Jahre grundlegende Menschenrechte verletzten, indem sie etwa das Folterverbot aushebelten, außerordentliche Überstellungen (rendition flights) durchführten und Menschen ohne Anklage und Gerichtsverfahren unter menschenunwürdigen Bedingungen in Haft hielten. ${ }^{57}$

Vielfältige aktuelle Herausforderungen an den Menschenrechtsschutz - von Terror und Terrorismusbekämpfung über Staatszerfall, Flucht und Migration bis hin zu Armut und der globalen Ernährungskrise - tragen auch hierzulande zur Wahrnehmung bei, dass die Menschenrechte insgesamt in die Defensive geraten sind. ${ }^{8}$ Doch sind in einigen menschenrechtlichen Bereichen, etwa bezüglich der wsk-Rechte, erhebliche Fortschritte erzielt worden, die es ebenso wie die Rückschritte differenziert zu analysieren gilt. Bei aller Wichtigkeit quantitativer Tendenzaussagen, bleibt die qualitative Analyse und Darstellung der Menschenrechtslage und -politik in und zu den jeweiligen Ländern unersetzlich.

56 Vgl. www.politicalterrorscale.org/comparisons.html (abgerufen am 29.12.2008).

57 Vgl. etwa den USA-Bericht des UN-Sonderberichterstatters zu Menschenrechten und Terrorismusbekämpfung (A/HRC/6/17/Add.3, 22.11.2007) sowie den gemeinsamen Guantánamo-Bericht mehrerer UN-Sonderberichterstatter(E/CN.4/2006/129, 27.02.2006).

58 So beispielsweise der Tenor vieler Beiträge in dem jährlich erscheinenden »Jahrbuch der Menschenrechte« oder dem Jahrbuch des Komitees für Grundrechte und Demokratie. 


\subsection{Die »Entkopplung«von Normanerkennung und Normeinbaltung}

Ausgehend von der allgemeinen Beobachtung, dass die Mehrheit der Staaten zwar wichtige Menschenrechtsabkommen ratifiziert hat, aber die Menschenrechte weiterhin weltweit verletzt werden, gehen verschiedene, teils quantitative Studien der Frage nach, inwieweit sich die Ratifizierung von Menschenrechtsabkommen auf die Implementierung und Einhaltung dieser Normen auswirkt. Die Befunde entsprechender Studien sind vorderhand ernüchternd, denn sie lassen keinen positiven statistischen Zusammenhang zwischen der Ratifikation von Menschenrechtsabkommen und der Einhaltung bzw. Lage der Menschenrechte erkennen, zumindest nicht bezogen auf autoritäre Staaten (und die jeweils untersuchten Rechte). ${ }^{59} \mathrm{Am}$ ehesten scheinen demokratische Staaten ihre menschenrechtlichen Selbstverpflichtungen ernst zu nehmen. Dies weist auf die enge, auch in quantitativen Studien festgestellte Verknüpfung zwischen Demokratie und Menschenrechten hin ${ }^{60}$, wenngleich die Befunde etwa nach Herrschaftssubtypen, nach Phasen des Übergangs oder nach Rechten zu differenzieren sind. So unterscheiden sich autoritäre Regime erheblich hinsichtlich ihres Repressionsniveaus, können demokratische Transitionen überaus konfliktiv sein und weist der Menschenrechtsschutz auch in Demokratien, zumal in jenen mit »illiberalen« Zügen, mitunter eine beachtliche Schieflage auf. ${ }^{61}$

Menschenrechtsabkommen allein verändern die Menschenrechtslage selbstredend nicht; sie sind aber auch unter autoritären Bedingungen nicht wertlos: Wenn Staaten die Menschenrechte völkerrechtlich anerkennen, können sie beim Wort genommen werden: Forderungen nach Einhaltung, Schutz und Umsetzung der Menschenrechte haben dadurch nicht nur eine moralische, sondern auch eine rechtliche Grundlage und entwickeln ggf. politische Schubkraft. Letzteres hängt maßgeblich davon ab, wie nachdrücklich diese Rechte eingefordert werden. So ergeben selbst die genannten quantitativen Studien von Neumayer und Hafner-Burton/Tsutsui, dass sich die Ratifikation von Menschenrechtsabkommen umso positiver auf die Menschenrechtslage auswirkt, je stärker die Zivilgesellschaft ist. ${ }^{62}$ Qualitative Studi-

59 Vgl. Eric Neumayer, »Do International Human Rights Treaties Improve Respect for Human Rights? in: Journal of Conflict Resolution, Vol. 49, Nr. 6 (2005), S. 925-953; Emilie M. Hafner-Burton / Kiyoteru Tsutsui, »Human Rights in a Globalizing World: The Paradox of Empty Promises in: American Journal of Sociology, Vol. 110, Nr. 5 (2005), S. 1371-1411; dies., Justice Lost! aaO. (Fn. 24).

60 Vgl. Emilie M. Hafner-Burton / James Ron, »Human Rights Institutions: Rhetoric and Efficacy « in: Journal of Peace Research, Vol. 44, Nr. 4 (2007), S. 379-383, hier: S. 380 f.

$61 \mathrm{Vgl}$. für Lateinamerika etwa: Michael Krennerich, »Menschenrechte in Lateinamerika. Schutz mit sozialer Schieflage« in: Brennpunkt Lateinamerika, Nr. 7 (2003), S. 57-68; Michael Krennerich / Manuel E. Góngora Mera, "Soziale Menschenrechte in Lateinamerika. Herausforderungen an Justiz, Politik und Wirtschaft « in: Brennpunkt Lateinamerika, Nr. 15 (2005), S. 173-188.

62 Vgl. Fn. 57 sowie: Anja Jetschke, »Weltkultur versus Partikularismus. Die Universalität der Menschenrechte im Lichte der Ratifikation von Menschenrechtsverträgen « in: Die Friedens-Warte, Jg. 81, H. 1 (2006), S. 25-49. 
en zu Ländern des Südens, die autoritär regiert werden oder Demokratisierungsprozesse durchliefen, belegen dies und weisen zudem auf die hohe Bedeutung transnationaler Menschenrechtsnetzwerke für die Durchsetzung internationaler Menschenrechtsnormen im Innern normverletzender Staaten hin. ${ }^{63}$ Internationale Menschenrechtsnormen haben demnach dann eine vergleichsweise große Chance auf Durchsetzung, wenn es transnationalen Menschenrechtsnetzwerken gelingt, die internationale Staatengemeinschaft und die innenpolitische Opposition bzw. Zivilgesellschaft zu mobilisieren und so die Regierung sowohl »von oben « als auch »von unten« unter Druck zu setzen. Nach dem sog. »Spiral-Modell« des Menschenrechtswandels führt dieser Druck zu einem mehrphasigen Reformprozess, der von Repression und Leugnen über taktische Konzessionen bis hin zu ernsthaften Reformen und normgeleitetem Verhalten seitens der Regierenden führen kann. ${ }^{64}$

Inwieweit sich solche Befunde auch auf die Durchsetzung weniger weit reichender Menschenrechtsreformen in etablierten westlichen Demokratien übertragen lassen, bleibt zu prüfen. Nur vereinzelt wurden auch westliche Demokratien, wie Israel oder Großbritannien, in entsprechende qualitative Untersuchungen einbezogen. ${ }^{65}$ Dabei ist zu bedenken, dass Menschenrechtsorganisationen und -netzwerke, gerade in einem menschenrechtsfreundlichen Umfeld, eben nicht nur »naming, shaming and blaming «-Strategien verfolgen, sondern auch Überzeugungs-, Lobby- und Advocacy-Arbeit betreiben. Dies leitet zur Frage über, wie die Umsetzung der Menschenrechte politisch befördert werden kann.

\subsection{Die Universalisierung der Menschenrechte als politisches Projekt}

Die bisherigen Ausführungen legen nahe, dass allgemein die Entwicklung von Demokratie und Rechtsstaatlichkeit sowie eine starke, international vernetzte Zivilgesellschaft günstige Voraussetzungen für die Umsetzung von Menschenrechten sind. Dies gilt nicht nur für die bürgerlich-politischen Rechte, die bereits definitorisch eng mit einem demokratischen Rechtsstaat verbunden sind, sondern auch für die wsk-Rechte. Ein Bericht der UN-Entwicklungsorganisation aus dem Jahre 2000 bezeichnet die Demokratie sogar als die einzige Regierungsform, die mit allen fünf Kategorien von Menschenrechten - den bürgerlichen, politischen, wirtschaftlichen,

63 Vgl. etwa Kathryn Sikkink, »Human Rights, Principled Issue-Networks, and Sovereignity in Latin America in: International Organization, Vol. 47, Nr. 3 (1993), S. 411-441; Ellen Lutz / Kathryn Sikkink, »International Human Rights and the Practice in Latin America «, in: International Organization, Vol. 54, Nr. 3 (2000), S. 633-659; Thomas Risse, "Menschenrechte als Grundlage der Weltvergemeinschaftung? Die Diskrepanz zwischen Normanerkennung und Normeinhaltung « in: Janz / Risse, Menschenrechte Globale Dimensionen eines universellen Anspruchs, aaO. (Fn. 29), S. 17-37.

64 Vgl. Thomas Risse / Anja Jetschke / Hans-Peter Schmitz, Die Macht der Menschenrechte: Internationale Normen, transnationale Netzwerke und innenpolitischer Wandel, Baden-Baden 2002.

65 Vgl. Andrea Liese, Staaten am Pranger. Zur Wirkung internationaler Regime auf innerstaatliche Menschenrechtspolitik, Wiesbaden 2006. 
sozialen und kulturellen Rechten - vereinbar sei. ${ }^{66}$ Im Falle der wsk-Rechte wäre allerdings darüber hinaus kritisch zu prüfen, inwieweit nicht nur die politische, sondern auch die wirtschaftliche und soziale Ordnung auf nationaler und internationaler Ebene menschenrechtskonform ausgestaltet ist. Hier wird von NGOs erheblicher Reformbedarf reklamiert. ${ }^{67}$

Die Politik ist daher angehalten, die übergreifenden politischen und sozioökonomischen Bedingungen, die der Umsetzung der Menschenrechte förderlich oder abträglich sind, in den Blick zu nehmen und im Rahmen ihrer Möglichkeiten mitzugestalten. Dabei liegt auf der Hand, dass entsprechende Veränderungsprozesse langwierig und die Einflussmöglichkeiten einzelner Staaten oder auch internationaler Organisationen begrenzt sind. Sowohl in der Entwicklungsländer- als auch in der Transitionsforschung wurde beispielsweise früh erkannt, wie schwierig es ist, »von außen« Demokratie und Menschenrechte in den jeweiligen Ländern zu befördern. ${ }^{68}$

Die Frage, was die Regierungen selbst dazu bewegen könnte, tatsächlich die Menschenrechte zu achten und umzusetzen, wird unterschiedlich beantwortet. Die Vorstellung freilich, dass repressive Staaten, wenn überhaupt, nur über $Z_{w a n g}$ und Druck dazu gebracht werden können, die Menschenrechte zu achten oder zumindest weniger stark zu verletzen, ist weit verbreitet und geht oft einher mit der Skepsis gegenüber dem internationalen Menschenrechtsregime, das über keine dem nationalen Recht vergleichbare Zwangsmittel verfügt. Diese Skepsis verbindet sich in der internationalen Theoriediskussion oft mit realistischen Positionen oder zumindest mit rationalistischen Sichtweisen. ${ }^{69}$ In das politische Handlungsschema passen hierzu politische, wirtschaftliche oder gar militärische Sanktionen gegenüber repressiven Staaten. ${ }^{70} \mathrm{Zu}$ letzteren gehören »humanitäre Interventionen « und die in jüngster Zeit diskutierte »responsiblity to protect « ${ }^{71}$ Nicht-militärische Sanktionen umfassen etwa die Einfrierung von Konten, Reisebeschränkungen, Waffen- und Handelembargos oder das (nicht unproblematische) Aussetzen von Hilfsprogrammen - Sanktionen also, welche etwa die Europäische Union vor allem gegenüber afrikanischen und einigen asiatischen Staaten schon angewandt hat. ${ }^{72}$

66 UNDP, Bericht über die menschliche Entwicklung 2000. Human Rights and Human Development, Bonn 2000, S. 69.

67 Statt vieler: Windfuhr, Beyond the Nation State, aaO. (Fn. 20).

68 Vgl. etwa Rolf Hanisch (Hg.), Demokratieexport in die Länder des Südens?, Hamburg 1996; Gero Erdmann, Demokratie- und Menschenrechtsförderung in der Dritten Welt, Bonn 1999; Christof Hartmann, Externe Faktoren im Demokratisierungsprozeß. Eine vergleichende Untersuchung afrikanischer Länder, Opladen 1999.

69 Vgl. Jetschke, Weltkultur versus Partikularismus, aaO. (Fn. 60), S. 36 ff.

70 Vgl. etwa: Richard B. Lillich et al., International Human Rights. Problems of Law, Policy, and Practice, New York 2006 (hier: Kap. 9).

71 Vgl. etwa Ramesh Thakur, »Humanitarian Intervention« in: Thomas G. Weiss / Sam Daws (Hg.), The Oxford Handbook on the United Nations, Oxford 2007, S. 387-403; Johannes Varwick, »Kämpfen für die Menschenrechte? Humanitäre Intervention und die Schutzverantwortung « in: Gareis / Geiger, Internationaler Schutz der Menschenrechte, aaO. (Fn. 37), S. 141-159. 
Auch diplomatischer Protest und öffentliche Kritik sind letztlich Druckmittel. Immerhin kann bereits die Veröffentlichung und das Anprangern staatlicher Menschenrechtsverletzungen im Sinne eines »Beschämens « und »Bedrängens « Wirkung entfalten, denn kaum eine Regierung der Welt möchte offen als Unrechtsregime dastehen. Diesen Umstand machen sich, wie bereits erwähnt, vor allem nicht-staatliche Menschenrechtsorganisationen zunutze. Aber auch die EU kritisiert beispielsweise in vertraulichen Demarchen oder öffentlichen Erklärungen Menschenrechtsverletzungen in Drittstaaten (oder lobt positive Entwicklungen) ${ }^{73}$ Und die politischen Organe des UN-Menschenrechtsschutz, wie der Menschenrechtsrat bzw. zuvor die Menschenrechtskommission, werden immer wieder für gegenseitige Kritik an Menschenrechtsverletzungen genutzt, leider oft zu wenig sachorientiert, sondern eingebettet in ein ausgeprägtes politisches Lagerdenken, was in der Vergangenheit zu selektivem Vorgehen, doppelten Standards und politisch motivierten Handlungsblockaden selbst bei schweren Menschenrechtsverletzungen führte. ${ }^{74}$

Im Sinne eines interessengeleiteten, rationalen Handels ist zudem anzunehmen, dass auch positive Anreize, etwa Wirtschaftshilfe oder die politische Unterstützung auf anderen Politikfeldern, das Kosten-Nutzen-Kalkül und damit das Verhalten der Staaten verändern können. Ein potenzieller EU-Beitritt ist beispielsweise ein solcher Anreiz. Ein anderer ergibt sich etwa durch Hilfsgelder. So hat sich gerade in der Entwicklungszusammenarbeit die Erkenntnis durchgesetzt, dass positive Maßnahmen und Anreize der Förderung der Menschenrechte Vorrang haben sollen vor Sanktionen, die dementsprechend nur als letztes Mittel gelten. Vielfältige Maßnahmen aktiver Menschenrechtsförderung zielen letztlich darauf ab, in den jeweiligen Ländern Prozesse zur Verbesserung der Menschenrechtslage voranzutreiben und solche Akteure, Organisationen und Institutionen direkt oder indirekt zu unterstützen, die als »drivers of change« in Erscheinung treten. Eine besondere Bedeutung kommt hierbei dem »empowerment « und der Partizipation gerade der benachteiligten und in ihren Rechten verletzten oder bedrohten Menschen zu. So zielt die Menschenrechtsförderung in der Entwicklungszusammenarbeit u.a. darauf ab, Menschen in die Lage zu versetzen und dabei zu unterstützen, ihre Rechte selbständig einzufordern, wahrzunehmen und zu überwachen und Entscheidungsprozesse aktiv mitzugestalten. ${ }^{75}$

Zudem handeln Regierungen nicht immer als »Nutzenmaximierer«. Mitunter übernehmen sie auch bestimmte Verhaltensweisen und institutionelle Vorgaben,

72 Vgl. Wolfgang Heinz / Josephine Liebl, »Wie tritt die EU im internationalen Bereich für die Menschenrechte ein? «, Nürnberg 2007 (Internetbeitrag, abrufbar unter: www.menschenrechte.org).

73 Vgl. die jährlichen EU-Jahresberichte zur Menschenrechtslage (http://europa.eu/pol/ rights/index_en.htm).

74 Vgl. Gunnar Theisen, »Mehr als nur ein Namenwechsel. Der neue Menschenrechtsrat der Vereinten Nationen « in: Vereinte Nationen, Nr. 4 (2006), S. 138-146; Theodor Rathgeber, "Beredtes Schweigen und schiefe Bahnen: Der Menschenrechtsrat der Vereinten Nationen« in: Heiner Bielefeldt et al. (Hg.), Jahrbuch Menschenrechte 2009, Wien u.a. 2008, sowie das kontinuierliche Monitoring des Menschenrechtsrates durch den International Service for Human Rights (www.ishr.ch).

75 Vgl. Michael Krennerich, Entwicklung und Menschenrechte, aaO. (Fn. 40). 
weil diese im internationalen Umfeld bereits bestehen oder als angemessen gelten. Die Anerkennung und Befolgung von Menschenrechtsnormen durch die jeweiligen Regierungen stellen sich nach dieser Lesart als eine sozialisations- und rollenbedingte Übernahme, Internalisierung oder Habitualisierung menschenrechtlicher Normen und Standards dar, die bereits in der » Weltkultur « verankert sind und der sich kein Staat legitimerweise entziehen kann, der zur Weltgemeinschaft gehört oder gehören will. Diese Annahmen verbinden sich mit einem soziologischen Institutionalismus ${ }^{76}$ und einer politischen Logik der Angemessenheit (anstatt der Zweckrationalität). ${ }^{77}$ Analoges gilt auf nationaler Ebene, wenn in einem Land bereits eine »Menschenrechtskultur besteht, die das Verhalten der politischen Akteure mitprägt. So wird gerade für lateinamerikanische - im Unterschied etwa zu asiatischen Staaten - die große Bedeutung einer liberalen Verfassungstradition und der kulturellen Einbettung der Menschenrechte hervorgehoben. ${ }^{7}$

Ferner sollte nicht in Abrede gestellt werden, dass Regierungen gerade menschenrechtsfreundlicher Staaten auch deswegen die Menschenrechte achten, weil eine solche Politik den grundlegenden Wertvorstellungen der Akteure entspricht. Dies beeinflusst auch das Verhalten nicht-staatlicher Menschenrechtsorganisationen. In Staaten mit einem vergleichsweise guten Menschenrechtsprofil verfolgen sie bezeichnenderweise nicht nur »naming, shaming and blaming «-Strategien gegenüber staatlichen Akteuren, sondern stehen auch mit »like-minded persons « in Regierung und Parlament im engen inhaltlichen Austausch, um Erfahrungen, Sichtweisen und Strategien der Menschenrechtspolitik abzustimmen, Überzeugungsarbeit zu leisten und ggf. Lernprozesse anzustoßen. Inwieweit es hierbei auch zu politischen Lernprozessen und einer Anpassung der nationalen Menschenrechtspolitiken an internationale Veränderungen und Herausforderungen kommt, bleibt im Einzelfall zu prüfen. Eine entsprechende theoriegeleitete, systematische Analyse der deutschen Menschenrechtspolitik steht diesbezüglich noch aus. ${ }^{79}$

Im Detail bleibt also zu untersuchen, inwiefern etwa Sanktionen, Druck, Anreize, Sozialisation und Überzeugungsarbeit tatsächlich Wirkung entfalten. Dies lässt sich aber nicht ohne systematische Bezugnahme auf die jeweiligen Bedingungen vor Ort klären. Denn die Staaten und Regierungen unterscheiden sich z.B. erheblich hinsichtlich ihrer militärischen, wirtschaftlichen, politischen oder auch moralischen »Verwundbarkeit«, und die beteiligten Akteure weisen ein unterschiedlich hohes $\mathrm{Maß}$ an Handlungsmöglichkeiten sowie an Lern- und Reformfähigkeit auf. Eine systematische Wirkungsanalyse von Menschenrechtspolitik und -förderung stellt

76 Vgl. Jetschke, Weltkultur versus Partikularismus, aaO. (Fn. 60), S. 40 ff.

77 Vgl. Liese, Staaten am Pranger, aaO. (Fn. 64), S. 44.

78 Paolo G. Carrazo, »From Conquest to Constitutions: Retrieving a Latin American Tradition of the Idea of Human Rights « in: Human Rights Quarterly, Vol. 25, Nr. 2 (2003), S. 281-313.

79 Politikwissenschaftlich lassen sich hier Überlegungen verschiedener Policy-Ansätze, etwa des »advocacy coalition-Ansatzes", des »lesson drawing «, des "policy transfers" oder auch der Bedeutung von »epistemic communities « auf die Menschenrechtspolitik übertragen - was aber bisher noch nicht geschehen ist. 
eine große, auch methodische Herausforderung für die Politik und die Politikwissenschaft dar.

\section{Zusammenfassung}

Menschenrechte sind komplexe Rechte. Sie lassen sich moralisch begründen und werden durch politische Entscheidungen zu positiven Rechten, die es wiederum umzusetzen gilt. Der Beitrag beschäftigt sich vornehmlich mit der rechtlichen wie politischen »Universalisierung « der Menschenrechte. Er zeigt auf, dass über die Ratifikation internationaler Menschenrechtsabkommen hinaus noch wichtige Aspekte der rechtlichen Anerkennung und Durchsetzung der Menschenrechte ausstehen und weist auf die hohe Bedeutung des nationalen Menschenrechtsschutzes hin. Vor dem Hintergrund der Bestandsaufnahme weltweiter Menschenrechtsverletzungen werden Demokratie, Rechtsstaatlichkeit und eine starke, international vernetzte $\mathrm{Zi}$ vilgesellschaft als wichtige Voraussetzungen für einen effektiven Menschenrechtsschutzes ausgewiesen und verschiedene Ansätze der Menschenrechtsförderung dargelegt.

\section{Abstract}

Human rights are complex rights. They can be morally justified, become positive rights by way of political decisions and must in turn be implemented. The article deals primarily with the legal as well as the political »universalisation« of human rights. It demonstrates that, going beyond the ratification of international human rights treaties there are other important aspects of the legal recognition and implementation of human rights still to be considered, and points out the importance of the protection of human rights on a national level. Against the background of a review of the situation as regards worldwide human rights violations democracy, the rule of law, and a strong, internationally linked civil society will be put forward as important prerequisites for an effective human rights protection. Further, a number of approaches to the promotion of human rights will be proposed.

Michael Krennerich, The universalisation of human rights - focus on the legal and political dimensions 